\title{
THE DYNAMIC BEHAVIOUR OF IONS IN FLUORITE AND TYSONITE-TYPE SOLID SOLUTIONS
}

\author{
J. SCHOONMAN \\ Solid State Department, Physics Laboratory, State University, P.O. Box 80.000, 3508 TA Utrecht, The Netherlands
}

\begin{abstract}
Metal fluorides with the fluorite $\left(\mathrm{CaF}_{2}\right)$ structure are known to accept large amounts of $\mathrm{LaF}_{3}$, rare earth (III) fluorides, $\mathrm{ThF}_{4}$, or $\mathrm{UF}_{4}$. On the other hand, metal fluorides with the tysonite $\left(\mathrm{LaF}_{3}\right)$ structure are known to dissolve large amounts of the alkaline earth fluorides. The electrical properties of these anion-excess fluorite-type, and anion-deficient tysonite-type solid solutions have attracted considerable attention in recent years. Nominally pure alkaline earth fluorides are rather poor anionic conductors, in contrast to the nominally pure tysonites. With regard to the composition dependence of the isothermal ionic conductivity, significant differences exist between both types of solid solution. The ionic conductivity of concentrated fluorite-type solid solutions increases exponentially with increasing solute content. In several of these systems a maximum is observed in the conductivity isotherm, while in all extensive defect clustering is assumed to occur. Up to about 5 mole \% a linear increase occurs in the tysonite-type solid solutions. Like the aniondeficient oxyfluorites, the conductivity isotherms reveal a maximum. In fluorites both fluoride ion vacancies, and fluoride interstitials carry the current. This has some unexpected consequences for the composition dependence of the ionic conductivity. In the tysonite-type solid solutions only fluoride ion vacancies are mobile. However, in this structure two types of anion lattice site need be taken into account. Recently, several conduction models have been proposed for both types of solid solution. These models will be discussed in relation with possible defect structures, which do not only affect the extrinsic conductivity, but in the case of the fluorite-type solid solutions also the high-temperature liquid-like conduction mode. The discussion will not be restricted to diffusive motion of the defects. In addition, it will be shown that the study of localized motion with thermal depolarization techniques can contribute significantly to our understanding of the conduction mechanisms of both types of solid solution.
\end{abstract}

\section{Discussion}

\section{J.B. Wagner:}

Please tell us how you obtained the specific type of defects present in the crystals from the thermally stimulated depolarization curves?

\section{J. Schoonman:}

The thermally stimulated depolarization curves do not provide information about the structure of the reorienting dipoles.

Detailed information about defect structures can be obtained from endor measurements, and EPR spectra.

\section{W.C. Mackrodt:}

Is the mechanism for dipolar relaxation you mentioned deduced from theory or can it be inferred from experiment in any way?

\section{J. Schoonman:}

The calculations of Chakravorty (1971) and of Catlow and Norgett (1973) show that the dominant mode of fluoride interstitial displacement is the noncollinear interstitialcy mechanism. This mechanism is assumed to also describe the motion of bound interstitials, like in the reorienting $\mathrm{nn}$ and $\mathrm{nnn}$ dipoles $\left(\operatorname{La}_{\mathbf{B a}} \mathrm{F}_{\mathrm{i}}\right)^{\times}$.

\section{C.R.A. Catlow:}

Have you considered the effect of dopant radius on conductivity? As neutron diffraction studies, that we have recently carried out, find that the cluster structure depends on the dopant radius.

\section{J. Schoonman:}

Defect clusters cause, irrespective of their complexity, lattice distortions. Fluoride interstitials move faster in a slightly distorted anion array, because $F$ lattice ions are involved in the non-colinear interstitialcy mechanism. The increase of the conductivity of the concentrated solid solutions is attributed to this mobility enhancement. The radius of the dopant need not be considered in detail. 